\title{
The patients with posterior reversible encephalopathy syndrome
}

\begin{abstract}
Posterior Reversible Encephalopathy Syndrome (PRES): is a syndrome that can develop due to eclampsia, preeclampsia, hypertension, drug intoxications and various metabolic diseases. It is a condition with symptoms such as visual impairment, headache, mental disorder, and epileptic seizures, which can improve clinically and radiologically within a few weeks with the elimination of the etiological factor. Vasogenic edema forming in cerebral tissue forms the basis of its pathophysiology.
\end{abstract}

Early diagnosis and treatment are of serious prognostic importance in PRES cases. Rapid regulation of blood pressure and immunosuppressive therapy should be prioritized.

Despite a wide variety of etiologies, pregnancy and postpartum are common causes for PRES. Selection of antihypertensive and antiepileptic drugs in early diagnosis and treatment and early initiation of anti-edema treatment reduce the rate of chronic neurological sequela in patients.

Keywords: pregnancy, eclampsia, preeclampsia, posterior reversible encephalopathy syndrome, PRES, anti epileptic, anti edema
Volume 12 Issue 3 - 2020

\author{
İpek Erus, ${ }^{2}$ Ahmet Akyol,' Pınar Yıldırım \\ Ozkan,' Ayșe Duygu Kavas,' Ahu Baysal \\ Çitil,' Ahmet Eroğlu ${ }^{3}$ \\ 'The University of Health Sciences, Ümraniye Training and \\ Research Hospital, Clinic of Anesthesiology and Intensive Care, \\ Turkey \\ ${ }^{2}$ Koc University, Medical Faculty, Clinic of Anesthesiology and \\ Intensive Care, Turkey \\ ${ }^{3} \mathrm{KTU}$, Medical Faculty, Clinic of Anesthesiology and Intensive \\ Care, Turkey
}

\begin{abstract}
Correspondence: Assoc. Prof. Dr.Ahmet AKYOL,The University of Health Sciences, Ümraniye Training and Research Hospital, Clinic of Anesthesiology and Intensive Care, Elmalikent Mah. Adem Yavuz Cad. No: I, (34764) Ümraniye, İstanbul, Turkey, Tel+90533633|369, Email ahmetakyol9598@gmail.com
\end{abstract}

Received: April 29, 2020 | Published: May 25, 2020

\section{Introduction and aim}

Posterior Reversible Encephalopathy Syndrome (PRES): is a syndrome that can develop due to eclampsia, preeclampsia, hypertension, drug intoxications and various metabolic diseases. It is a condition with symptoms such as visual impairment, headache, mental disorder, and epileptic seizures, which can improve clinically and radiologically within a few weeks with the elimination of the etiological factor. Vasogenic edema forming in cerebral tissue forms the basis of its pathophysiology. Leakage in cerebral arterioles or vascular endothelial damage due to hyperperfusion have been blamed as the cause of vasogenic edema. Its etiology includes hypertensive encephalopathy, preeclampsia, acute and chronic kidney diseases, immunosuppressive drugs, blood transfusion and electrolyte imbalance. ${ }^{1-6}$

In this case, we aimed to evaluate our patient, who previously exhibited no risk factor, presenting with status epilepticus at the 30th week of pregnancy and diagnosed with PRES upon clinical and neuroradiological findings, in the light of the literature.

\section{Clinical case}

The patient, 27-year-old and 30-week pregnant based on the last menstrual period, had a generalized tonic-clonic seizure at home and was admitted to our emergency service with an ambulance. In the initial examination, she had the following values: BP: $170 / 100 \mathrm{mmHg}$ pulse: 120/min, $\mathrm{SpO}_{2}$ : 90\%, GCS: 6 (E:2 M:3 V:1). The patient was administered diazepam and magnesium bolus intravenously, then infusion was performed. A cesarean section was indicated by the obstetrics and gynecology clinic and the patient was transferred to the operating room with the airways kept open receiving oxygen support though a mask, with the following values; BP: $197 / 122 \mathrm{mmHg}$, pulse: $120, \mathrm{SpO}_{2}: 93 \%$. The orotracheal intubation was performed with rapid sequence induction. At the second minute into the operation following the extraction of the fetus, the values were as follows: BP: $100 / 65 \mathrm{mmHg}$ pulse: $125 / \mathrm{min}, \mathrm{SpO}_{2}: 100 \%$, pupillary isochoric IR $(+/+)$. The cranial MR was performed, and the patient was taken to the intensive care unit postoperatively, with orotracheal intubation and sedation. MRI showed subcortical hyperintensities around both fronto-parieto-occipital areas, bilateral basal ganglia and bilateral cerebellar hemispheres in the flair sequences, and vasogenic edema in the diffusion-weighted sequences. Millimetric areas of acute lacunar ischemia were observed in the right periventricular and deep white matter and in the right globus pallidus. Radiological and clinical findings were consulted with the neurologists and the patient was diagnosed with PRES. The patient was sedated by remifentanil and midazolam infusion (orotracheal intubated) and monitored. Intravenous infusion of magnesium was continued. 2X500 $\mathrm{mg}$ of intravenous Levetiracetam and 4X4mg of Dekort were preferred.

The liver and kidney function tests, hemoglobin hematocrit, platelet count, bleeding diathesis and proteinuria were found within normal limits. Control MRI revealed no additional pathology on postoperative day 1 .

The patient was monitored sedated and intubated for 72 hours. Then, sedative agents were gradually cut back and the patient was extubated. Hypertension developing during extubation was controlled by intravenous brevibloc infusion, and oral administration of 4X4 $\mathrm{mg}$ of alpha-adrenergic receptor antagonist was started. Patient was conscious, exhibited limited cooperation and no deficiency was observed in the neurological examination.

\section{Discussion}

The pathophysiology of PRES has not been precisely defined. Two possible hypotheses are proposed. The first is vasospasm caused by the overreaction of brain autoregulation resulting in potentially reversible ischemia to the brain in the vascular border 
zone territories. Ischemia and cytotoxic edema are believed to occur due to cerebral vasoconstriction with the stimulation of sympathetic system following acute hypertension attacks. However, many cases present with no vasospasm in large veins. Although brain perfusion SPECT scans show hypoperfusion in some cases, most present with hyperperfusion. ${ }^{7}$

The second theory, hyperperfusion theory, which explains the pathophysiology of PRES, is more widely accepted. Homeostatic mechanisms try to provide constant blood flow to the brain by autoregulation. Autoregulation thresholds tend to shift further down with decreased systemic blood pressure. Distal arterioles become dilated to increase blood flow to the brain. On the other hand, autoregulation threshold tends to increase with increasing systemic blood pressure. Cerebral arterioles contract to increase resistance and block blood flow to the brain and hence inhibit hyperperfusion. There is a defined upper limit for cerebral autoregulation in excessive spontaneous increases of blood pressure demonstrated by animal models. When exceeded, the arterioles cannot contract anymore and are forced to dilate with increased blood pressure. This is firstly observed in small segments of the arterioles, then spreads to the entire vein. ${ }^{8}$ When the threshold is exceeded, the perfusion pressure crosses the blood-brain barrier resulting in the extravasation of fluid, macromolecules, and even erythrocytes into the brain parenchyma. ${ }^{8}$ Firm and organized structure of the cerebral cortex shows resistance against the formation of excessive edema. Edema tends to spread to the subcortical white matter with continued breakdown of the bloodbrain barrier. ${ }^{9}$ Edema is gradually cleared from the subcortical white matter with the recovery of blood-brain barrier. Autoregulation is also partially affected by the sympathetic system that innervates the muscular walls of the cerebral arteries. The sympathetic system is known to be weaker in posterior circulation than anterior circulation. ${ }^{10}$ This helps explain the relatively posterior distribution of radiological imaging findings seen in PRES. It is characterized by the presence of bilateral cortical-subcortical hyperintense lesions in posterior cerebral regions on the FLAIR and T2-weighted sequences in cranial MRI. ${ }^{2-4,6}$ In advanced cases, lesions can be observed in the brainstem, cerebellum, basal ganglia and frontal lobes. ${ }^{11}$ In our case, cranial imaging revealed lesions and vasogenic edema in both fronto-parieto-occipital areas, bilateral basal ganglia and bilateral cerebellar hemispheres, indicating a high degree of disturbance in cerebral autoregulation.

Striano et al. conducted a study where they examined 8 cases developing postpartum PRES and reported that 5 of the cases were diagnosed with eclampsia while the other 3 had no predisposing factor accompanying pregnancy, as in our case. Prednisone and cyclophosphamide were initiated for treatment after the diagnosis was radiologically confirmed with MRI. To regulate blood pressure, they preferred calcium channel blockers as antihypertensives and used phenobarbital, sodium valproate, magnesium sulfate and benzodiazepine as antiepileptics. ${ }^{12}$

Akgun N. et al. examined two cases developing postpartum PRES. Following normal spontaneous delivery in the last trimester of pregnancy, without a predisposing factor, the patient had convulsion on day 8 , which was detected to be accompanied by hypertension and proteinuria. Diagnosis was confirmed by cranial CT. They used diazepam, magnesium sulfate and phenytoin as antiepileptic drugs, methyldopa as an antihypertensive, and planned antiedema therapy.

The second case presented with sudden headache and visual impairment at the 27th week of pregnancy. Hypertension and proteinuria were detected in the tests while the initial examination revealed visual impairment, anisocoria and light reflex weakness and the funduscopic examination revealed bilateral papillary edema and hypertensive retinopathy. The patient was diagnosed upon the detection of mild vasogenic edema on abdominal CT and bilateral ischemic areas extending from vertex to cerebellum on MRI. After emergency cesarean section, the patient sedated with midazolam underwent orotracheal intubation and monitored in the ICU, supported by a mechanical ventilator. Dexamethasone $(4 \mathrm{x} 8 \mathrm{mg})$, phenytoin $(15 \mathrm{mg} / \mathrm{kg}$ loading dose and $5 \mathrm{mg} / \mathrm{kg}$ maintenance), Norvasc $5 \mathrm{mg}$ (2x1), Perlinganit (10 $\mathrm{mcg} / \mathrm{kg} /$ hour) and fluid treatment with high oncotic pressure were started. $\mathrm{Mg} 4 \mathrm{~g}$ bolus was administered and then maintained at a dose of $2 \mathrm{~g} /$ hour. $^{13}$

Early diagnosis and treatment are of serious prognostic importance in PRES cases. Rapid regulation of blood pressure and immunosuppressive therapy should be prioritized. Nicardipine or labetalol are first-choice agents. ACE inhibitors should be avoided in obstetric patients due to their toxic effects on fetal kidney. ${ }^{14,15}$ Nitroglycerin has also been reported to possibly aggregate edema by causing cerebral vasodilation in these patients. ${ }^{16}$ Perioral nimodipine and calcium-channel blockers (against cerebral vasospasm) should be preferred in this patient group. The putative neuroprotective effect of nimodipine should also be kept in mind. ${ }^{17}$ In critical patients, EEG monitoring should be performed if possible and brain function should be monitored in terms of status epilepticus and cerebral ischemia. Magnesium sulfate therapy is the gold standard for eclamptic seizures, especially in pregnant women with preeclampsia. ${ }^{18}$ Benzodiazepine, a respiratory depressant, and phenytoin and cardiophenytoin, which have cardiac side effects, should be approached with caution in this group of patients. ${ }^{15}$

\section{Conclusion}

Despite a wide variety of etiologies, pregnancy and postpartum are common causes for PRES. Selection of antihypertensive and antiepileptic drugs in early diagnosis and treatment and early initiation of anti-edema treatment reduce the rate of chronic neurological sequela in patients.

\section{Authors' contributions}

$\mathrm{AA}$ and $\mathrm{AE}$ designed the study and drafted the manuscript. IE, PYÖ, ADK, ABÇ and AA collected the clinical and laboratory data. All authors read and approved the final manuscript.

\section{Conflicts of interest}

The authors declare that there is no conflict of interest regarding the publication of this paper.

\section{References}

1. Hinchey J, Chaves C, Appignani B, et al. A reversible posterior leukoencephalopathy syndrome. N Engl J Med. 1996;334(8):494-500.

2. Ringelstein EB, Knecht S. Cerebral small vessel diseases: manifestations in young women. Curr Opin Neurol. 2006;19(1):55-62.

3. Teksam M CSO, Michel E, Truwit CL. Posterior reversibl ensefalopati sendromu: patofizyoloji ve ileri MRG teknikleri ile korelasyon. Tanisal ve Girişimsel Radyoloji. 2001;464-472.

4. Demirtafl Ö GF, Vidinli B. Preeklampsi ve Eklampside Kranial MR Görüntüleme ve Klinik Korelasyon. Diag Interv Radiol. 2005;189-194. 
5. Pande AR, Ando K, Ishikura R, et al. Clinicoradiological factors influencing the reversibility of posterior reversible encephalopathy syndrome: a multicenter study. Radiat Med. 2006;24(10):659-668.

6. Yano Y, Kario K, Fukunaga T, et al. A case of reversible posterior leukoencephalopathy syndrome caused by transient hypercoagulable state induced by infection. Hypertens Res. 2005;28(7):619-623.

7. Szer IS, Miller JH, Rawlings D, et al. Cerebral perfusion abnormalities in children with central nervous system manifestations of lupus detected by single photon emission computed tomography. $J$ Rheumatol. 1993;20(12):2143-2148.

8. MacKenzie ET, Strandgaard S, Graham DI, et al. Effects of acutely induced hypertension in cats on pial arteriolar caliber, local cerebral blood flow, and the blood-brain barrier. Circ Res. 1976;39(1):33-41.

9. Kalimo H, Fredriksson K, Nordborg C, et al. The spread of brain oedema in hypertensive brain injury. Med Biol. 1986;64(2-3):133-137.

10. Edvinsson L, Owman C, Sjoberg NO. Autonomic nerves, mast cells, and amine receptors in human brain vessels. A histochemical and pharmacological study. Brain Res. 1976;115(3):377-393.

11. Narbone MC, Musolino R, Granata F, et al. PRES: posterior or potentially reversible encephalopathy syndrome? Neurol Sci. 2006;27(3):187-189.

12. Striano P, Striano S, Tortora F, et al. Clinical spectrum and critical care management of Posterior Reversible Encephalopathy Syndrome (PRES). Med Sci Monit. 2005;11(11):CR549-53.
13. Nur AKGÜN MK, Selma BASYİGİT, Harun YILMAZ, et al. Posterior Reversbl Ensefalopati: 2 Olgu Sunumu. İstanbul Tip Dergisi. 2010 2:82-85.

14. Kaplan PW. Neurologic aspects of eclampsia. Neurol Clin. 2004;22(4):841-861

15. Mednick AS, Mayer SA. Critical care management of neurologic catastrophes. Adv Neurol. 2002;90:87-101.

16. Finsterer J, Schlager T, Kopsa W, et al. Nitroglycerin-aggravated pre-eclamptic posterior reversible encephalopathy syndrome (PRES). Neurology. 2003;61(5):715-716.

17. Harkany T, Dijkstra IM, Oosterink BJ, et al. Increased amyloid precursor protein expression and serotonergic sprouting following excitotoxic lesion of the rat magnocellular nucleus basalis: neuroprotection by $\mathrm{Ca}(2+)$ antagonist nimodipine. Neuroscience. 2000;101(1):101-114.

18. Altman D, Carroli G, Duley L, et al. Do women with pre-eclampsia, and their babies, benefit from magnesium sulphate? The Magpie Trial: a randomised placebo-controlled trial. Lancet. 2002;359(9321):18771890 . 